\title{
O impacto da pandemia do SARS-CoV-2 nas doenças cardiovasculares
}

\author{
The impact of the SARS-CoV-2 pandemic on cardiovascular diseases \\ El impacto de la pandemia de SARS-CoV-2 en las enfermedades cardiovasculares
}

Claudicéia Francisca Noleto da Conceição ORCID: https://orcid.org/0000-0002-3881-4320 Hospital Getúlio Vargas, Brasil E-mail: claudiceianoleto@hotmail.com

José Chagas Pinheiro Neto

ORCID: https://orcid.org/0000-0002-6626-6122 Universidade Federal do Piauí, Brasil E-mail: jose.pinheiro4321@gmail.com

Higo José Neri da Silva ORCID: https://orcid.org/0000-0003-2089-4147 Universidade Federal do Piauí, Brasil E-mail: higoneri@gmail.com

Ana Carolina Hortencio Garcia

ORCID: https://orcid.org/0000-0001-5153-254X Centro Universitário de Brasília, Brasil E-mail: carolinahortencio@gmail.com

Denys Wanderson Pereira Frazão ORCID: https://orcid.org/0000-0002-6527-0912 IPR Cursos e Engenharia Clínica, Brasil E-mail: denysfrazao@gmail.com

Danilo do Nascimento Viana ORCID: https://orcid.org/0000-0002-0049-0383 Centro Universitário UNINOVAFAPI, Brasil E-mail: danilonviana@gmail.com

Alexandre Mendes Soares

ORCID: https://orcid.org/0000-0002-9291-0452 Centro Universitário UNINOVAFAPI, Brasil

E-mail: alexandresoares.bio@hotmail.com

Lizandro de Freitas Pessoa

ORCID: https://orcid.org/0000-0001-6941-6547

Centro Universitário UNIFACEMA, Brasil

E-mail: lizandrofreitas23@hotmail.com

Denis Romulo Leite Furtado

ORCID: https://orcid.org/0000-0002-6266-1061

Laboratório Central do Piauí, Brasil E-mail: kelly-rego@bol.com.br

Lucas de Oliveira Sousa

ORCID: https://orcid.org/0000-0001-8940-5597

Centro Universitário UNINASSAU, Brasil

E-mail: luccas.oliveira@ymail.com

Pétterson Danilo de Oliveira Lima Goiano ORCID: https://orcid.org/0000-0003-3630-8210

Universidade Federal do Piauí, Brasil

E-mail: danilogoianoenf@outlook.com

Ednaldo Francisco Santos Oliveira Junior

ORCID: https://orcid.org/0000-0003-2396-7655

Instituto Federal do Piauí, Brasil

E-mail: ednaldojunior.oliveira31@gmail.com

Ian Jhemes Oliveira Sousa

ORCID: https://orcid.org/0000-0003-1930-9828

Faculdade de Tecnologia de Teresina, Brasil

Universidade Federal do Piauí, Brasil E-mail: ianjhemes@gmail.com

Rodrigo Lopes Gomes Gonçalves

ORCID: https://orcid.org/0000-0001-6482-4998

Universidade Federal do Piauí, Brasil

Faculdade de Tecnologia de Teresina, Brasil

E-mail:profrodrigolgoncalves@gmail.com

Kelly Maria Rêgo Da Silva

ORCID: https://orcid.org/0000-0003-3070-4060

Laboratório Central do Piauí, Brasil

E-mail: kellyma.aria@gmail.com 


\begin{abstract}
Resumo
De acordo com pesquisas realizadas na China, o local de origem da pandemia, as lesões cardíacas se tornaram características desse novo vírus, chegando a cometer cerca de 30\% dos pacientes e levando a óbitos $40 \%$ deles. Diversas alterações foram observadas como: lesão miocárdica (20\% dos casos), arritmias (16\%), miocardite (10\%), além de insuficiência cardíaca (IC) e choque (até 5\% dos casos). O objetivo desse trabalho é informar e descrever sobre o aumento de doenças cardiovasculares ocasionadas pelo novo vírus. E mostrar a importância de mais estudos sobre a área. Os dados foram coletados utilizando as bases científicas: Literatura Latino-Americana e do Caribe em Ciências da Saúde (LILACS), National Library of medicine (PUBMED), PERIODICOS, SCIENCE DIRECT, Biblioteca Virtual em saúde - (BVS), Scientific Eletronic Library Online (SCIELO), Cochrane Library, HighWire Press, Scopus e Elsevier. Foram encontrados aproximadamente 600 artigos, foram verificados os idiomas, e se realmente se tratava sobre a correlação de problemas cardiovasculares e o novo vírus, então foram obtidos 39 artigos para a realização da discussão. Esse trabalho conclui que de acordo com as pesquisas existe uma grande correlação do vírus com as doenças cardiovasculares e lesões miocárdica, por conta do aumento da troponina, levando os pacientes a complicações maiores. Isso ocorre também por conta do aumento dos marcadores levando a lesões cardiovasculares.

Palavras-chave: Miocárdio; Saúde; Novo coronavírus.
\end{abstract}

\begin{abstract}
According to research carried out in China, the place of origin of the pandemic, cardiac injuries have become characteristic of this new virus, reaching about $30 \%$ of patients and leading to $40 \%$ of deaths. Several changes were observed such as: myocardial injury (20\% of cases), arrhythmias (16\%), myocarditis (10\%), in addition to heart failure (HF) and shock (up to 5\% of cases). The objective of this work is to inform and describe the increase in cardiovascular diseases caused by the new virus. And show the importance of more studies on the area. Data were collected using the scientific bases: Latin American and Caribbean Literature in Health Sciences (LILACS), National Library of medicine (PUBMED), PERIODICOS, SCIENCE DIRECT, Virtual Health Library - (BVS), Scientific Eletronic Library Online (SCIELO), Cochrane Library, HighWire Press, Scopus and Elsevier. Approximately 600 articles were found, languages were checked, and if it was really about the correlation of cardiovascular problems and the new virus, then 39 articles were obtained for the discussion. This work concludes that, according to research, there is a great correlation between the virus and cardiovascular diseases and myocardial injuries, due to the increase in troponin, leading patients to major complications. This is also due to the increase in markers leading to cardiovascular injuries.
\end{abstract}

Keywords: Myocardium; Health; New coronavirus.

\begin{abstract}
Resumen
Según una investigación realizada en China, lugar de origen de la pandemia, las lesiones cardíacas se han convertido en características de este nuevo virus, alcanzando alrededor del 30\% de los pacientes y provocando el $40 \%$ de las muertes. Se observaron varios cambios: lesión miocárdica (20\% de los casos), arritmias (16\%), miocarditis (10\%), además de insuficiencia cardíaca (IC) y shock (hasta un 5\% de los casos). El objetivo de este trabajo es informar y describir el aumento de enfermedades cardiovasculares provocadas por el nuevo virus. Y mostrar la importancia de más estudios sobre la zona. Los datos fueron recolectados utilizando las bases científicas: Literatura Latinoamericana y del Caribe en Ciencias de la Salud (LILACS), Biblioteca Nacional de Medicina (PUBMED), PERIODICOS, SCIENCE DIRECT, Biblioteca Virtual en Salud - (BVS), Scientific Eletronic Library Online (SCIELO), Cochrane Library, HighWire Press, Scopus y Elsevier. Se encontraron aproximadamente 600 artículos, se revisaron los idiomas y si realmente se trataba de la correlación entre los problemas cardiovasculares y el nuevo virus, se obtuvieron 39 artículos para la discusión. Este trabajo concluye que, según la investigación, existe una gran correlación entre el virus y las enfermedades cardiovasculares y las lesiones miocárdicas, debido al aumento de la troponina, lo que lleva a los pacientes a complicaciones mayores. Esto también se debe al aumento de marcadores que conducen a lesiones cardiovasculares.
\end{abstract}

Palabras clave: Miocardio; Salud; Nuevo coronavirus.

\title{
1. Introdução
}

O sistema cardiovascular em equilíbrio é o que propicia a eficácia de mecanismos como a frequência cardíaca e a pressão arterial. Diversas patologias e fatores de riscos facilitam vários problemas cardiovasculares (diabetes, obesidade, hipertensão, idade, dentre outros) (Fang, Karakiulakis \& Roth, 2020).

A mudança de rotina, alimentação, a utilização de tabaco, empregos, estudos e a prática de exercícios físicos ou fatores emocionais facilitam para a complicação da doença (Kim et al., 2020).

Os maus hábitos alimentares, rotina e atividades físicas, o aumento desses hábitos pode ocasionar esses fatores de 
risco como a diabetes e colesterol, que afetam diretamente os problemas cardiovasculares e outras doenças (Million, 2020).

De acordo com pesquisas realizadas na China, o local de origem da pandemia, as lesões cardíacas se tornaram características desse novo vírus, chegando a cometer cerca de 30\% dos pacientes e levando a óbitos 40\% deles. Diversas alterações foram observadas como: lesão miocárdica (20\% dos casos), arritmias (16\%), miocardite (10\%), além de insuficiência cardíaca (IC) e choque (até 5\% dos casos) (Zheng et al., 2020).

O objetivo desse trabalho é informar e descrever sobre o aumento de doenças cardiovasculares ocasionadas pelo novo vírus. E mostrar a importância de mais estudos sobre a área.

\section{Metodologia}

Trata-se de uma pesquisa documental, de objetivo descritivo e explicativo com abordagem qualitativa e quantitativa. Essa pesquisa buscou através de publicações cientificas descrever seus resultados, explicando suas causas e efeitos. Sua abordagem implica que tudo o que foi realizado foi qualificado, quantificado e tabelado para melhor demonstração dos resultados obtidos pela pesquisa (Kauark, 2010).

Foram utilizadas todas as publicações que possuírem dados sobre a correlação de problemas cardiovasculares e o novo SARS-CoV-2, publicações cientificas, oficiais da Organização Mundial da Saúde (World Health Organization - WHO) e governamentais, dentre a determinada data (2019-2021).

Os dados foram coletados utilizando as bases cientificas: Literatura Latino-Americana e do Caribe em Ciências da Saúde (LILACS), National Library of medicine (PUBMED), PERIODICOS, SCIENCE DIRECT, Biblioteca Virtual em saúde - (BVS), Scientific Eletronic Library Online (SCIELO), Cochrane Library, HighWire Press, Scopus e Elsevier.

Foram analisados os trabalhos a nível mundial, nacional e estadual; e comparados para maior relevância dos resultados. Enaltecendo os resultados trabalhados.

Foram utilizados nessa pesquisa todos os trabalhos que relatam sobre a correlação de problemas cardiovasculares e o Novo Coronavírus, publicados nos anos de 2019 e 2021, nos idiomas: inglês, espanhol e português. Foram excluídos todos os demais trabalhos que não trataram do Novo Coronavírus, e publicações em outros idiomas não citados a cima.

A pesquisa não trouxe nenhum risco, pois todos os dados foram ofertados pelas bases cientificas, onde não houve contato nenhum com os indivíduos infectados e não foram divulgados dados pessoais dos mesmos. A pesquisa se deu apenas por análise de dados para demonstração do assunto tratado.

\section{Resultados e Discussão}

Foram encontrados aproximadamente 600 artigos, foram verificados os idiomas, e se realmente se tratava sobre a correlação de problemas cardiovasculares e o novo vírus, então foram obtidos 39 artigos para a realização da discussão.

\subsection{COVID-19: uma pandemia que alerta à população}

No ano de 2019 na cidade de Wuhan foi registrado o primeiro caso de uma mutação do Coronavírus o SARS-CoV-2, desde então em poucos meses se tornou uma pandemia atingindo mais de 27 países (11 de março de 2019) (OPAS, 2020).

Na Figura 1 podemos ver uma ilustração que representa a forma do Novo Coronavírus. 
Figura 1: Forma do Novo Coronavírus.

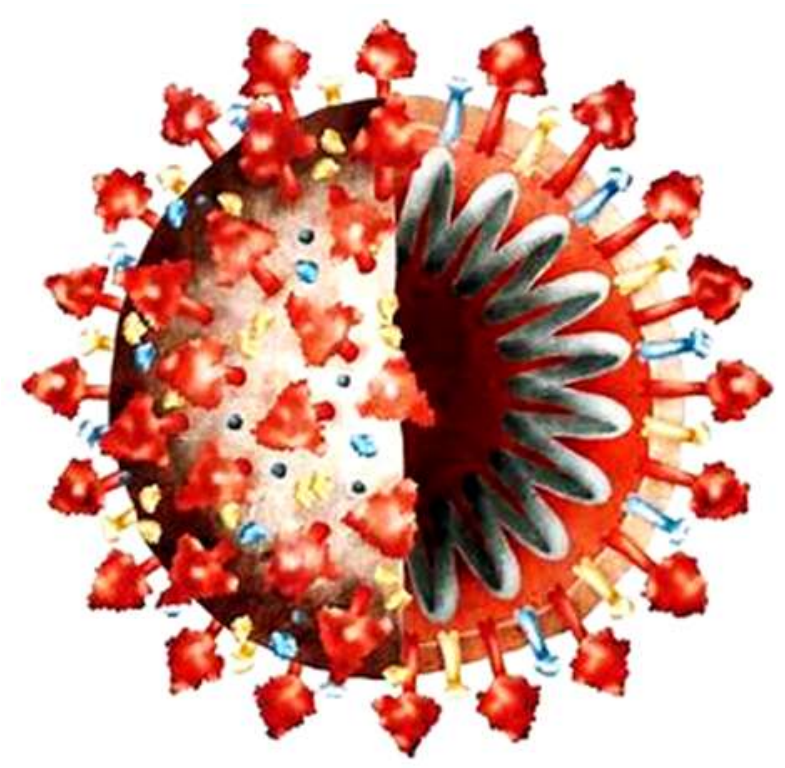

Fonte: Google imagens (2021).

Segundo a Organização Mundial de Saúde atualmente são 94.702.284 casos confirmados com 2.025.474 mortes no mundo sendo que 8.455.059 casos confirmados e 209.296 mortes apenas no Brasil. Com esses números o Brasil ocupa o terceiro lugar em número de casos confirmados (OPAS, 2020).

Esse aumento em números de casos ocorre por ser um vírus que atinge o trato respiratório com fácil proliferação. Outro motivo também acontece pela presença de mutações do vírus, possuindo hoje a presença de três cepas já identificadas. Esse vírus causa dificuldade de respirar, febre, diarreia, ausência do olfato e paladar, dor no corpo, dentre outros. O novo coronavírus também pode levar a complicações como pneumonia e sequelas até perda do funcionamento dos pulmões, síndromes ou miocardite (Driggin et al., 2020).

De acordo com algumas publicações, o SARS-CoV-2 se liga às células alvo dos hospedeiros através do domínio peptidase da enzima conversora de angiotensina 2 (ECA2), o que facilita a sua entrada e replicação (Gautret et al., 2020).

$\mathrm{O}$ vírus é da ordem dos Nidovirales da família Coronaviridae. Os morcegos-ferradura chineses funcionam como reservatório do coronavírus, no entanto ainda não se sabe a origem certa do vírus, a segunda cepa foi isolada na Inglaterra e a terceira no Brasil no estado do Amazonas (FIOCRUZ, 2020).

A maioria dos estudos revela que o vírus afeta mais os grupos de risco como diabéticos, pacientes com hipertensão, idosos, por que utilizam medicamentos inibidores da ECA facilitando a entrada do patógeno. Atualmente ainda não possui tratamento especifico, ainda existem muitos fármacos sendo estudados. Possui atualmente 28 vacinas sendo pesquisadas e já existem vacina liberadas em vários países, inclusive duas já sendo utilizadas no Brasil (Fang, Karakiulakis \& Roth, 2020).

\subsection{Anatomia e fisiologia do sistema cardiovascular}

O coração é responsável pelo bombeamento do sangue para todo corpo, ele possui o sistema nervoso simpático e parassimpático, elas são influenciadas por quimiorreceptores, barorreceptores e a participação de outros sistemas como o sistema respiratório, nervoso, vasomotor, renina-angiotensina-aldosterona e o termorregulador (Guyton \& Hall, 2006).

Demonstração da anatomia do coração humano representado pela ilustração na Figura 2. 


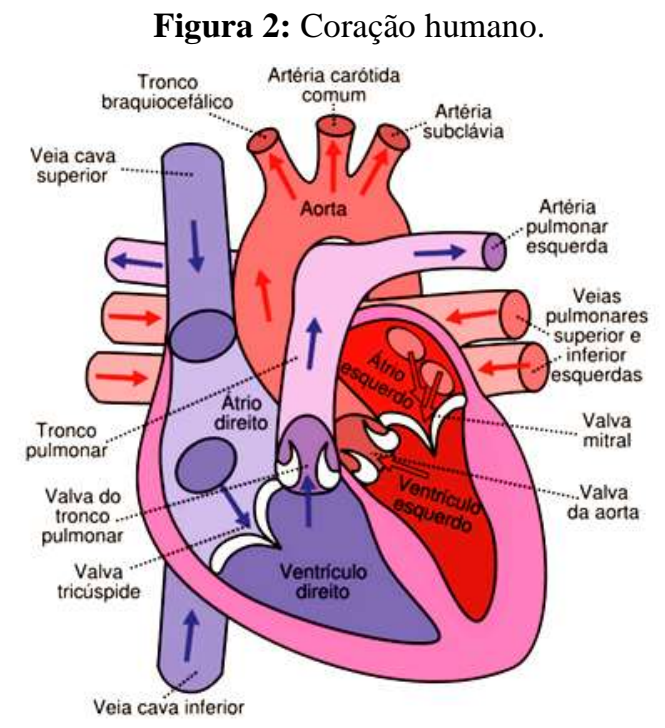

Fonte: Google imagens (2021).

A frequência cardíaca depende de uma variação complexa para gerar a regulação dos batimentos e suas mudanças, para que tudo ocorra dentro na normalidade (Marães et al., 2005).

O sistema parassimpático é predominante quando se trata do nódulo sinoatrial já o simpático sobre o nódulo atrioventricular, entretanto as vias nas conexões do sistema nervoso central e o controle do sistema cardiovascular os nervos vagos levam impulsos para os nódulos sinoatrial e atrioventricular e para os átrios atingindo também os ventrículos, onde ainda não se tem explicação exata com ocorre (Junqueira, 1990).

Hon e Lee (1965) foram os primeiros a apresentar informações sobre a variação da frequência cardíaca, através do sistema simpático e parassimpático. Utilizando a observação no sofrimento fetal simultâneo aos batimentos demonstrando distúrbios cardíacos.

Já Ewing et al. (1985) nos anos 70, estudaram pacientes com diabetes com variação nos graus de disautonomia no sistema cardíaco, o que diminuía a frequência cardíaca. Comparando a frequência cardíaca com a mortalidade em pacientes pós-infartados.

A idade também modifica a anatomia cardíaca, como a diminuição do tônus vagal e a redução da frequência cardíaca, o que também ocorre no momento de repouso (Guyton \& Hall, 2006).

Estudos mostram que o acompanhamento e análise de casos ou até mesmo a avaliação genética influenciam na redução de complicações cardiovasculares e funcionam como maior auxilio para um excelente tratamento (Gamelin, Berthoin \& Bosquet, 2006).

Na Figura 3 podemos analisar uma ilustração da fisiologia do sistema cardiovascular humano. 
Figura 3: Fisiologia do sistema cardiovascular.

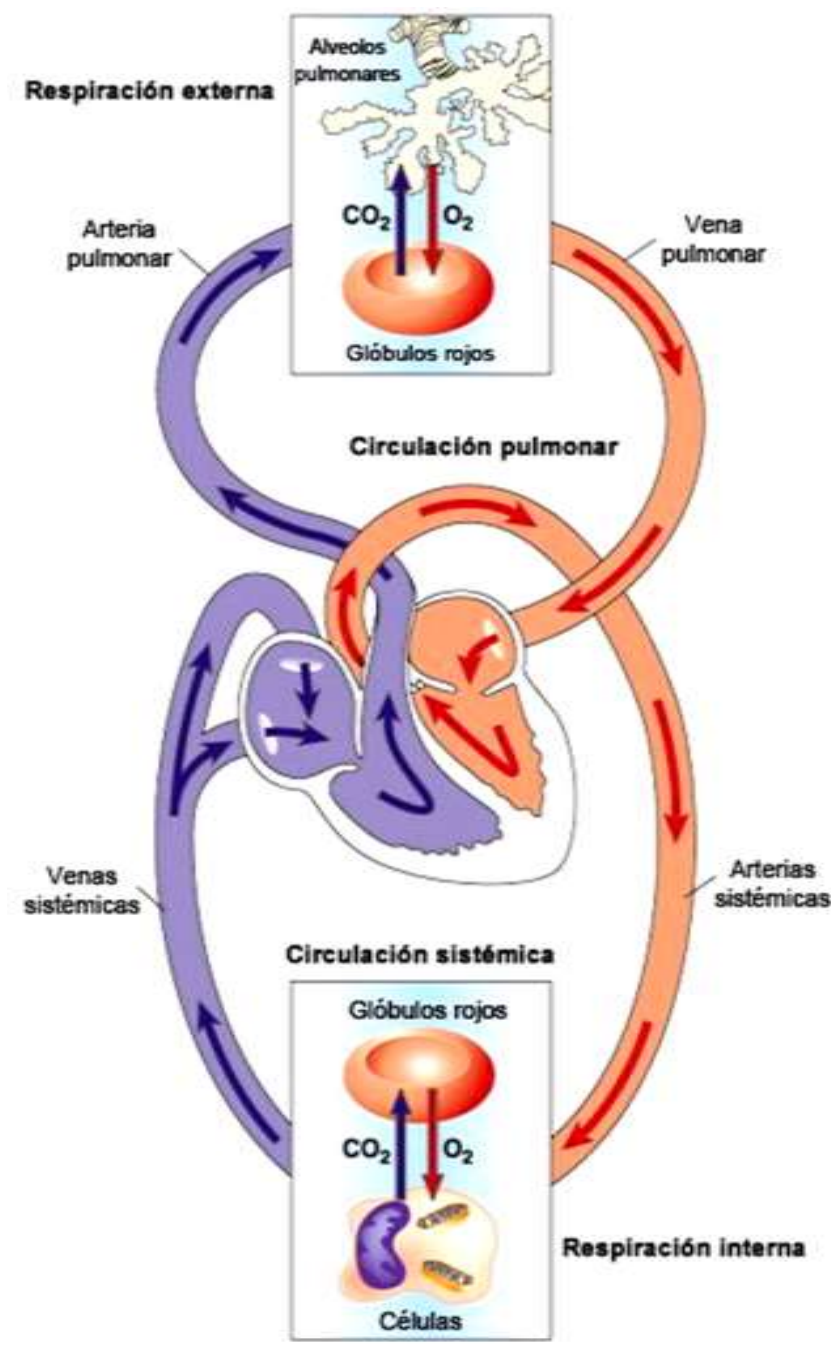

Fonte: Google imagens (2021).

O equilíbrio do sistema cardiovascular é realizado por diversos mecanismos, o que mantém o controle da pressão arterial e volume venoso (Leon, Shaver \& Leonard, 1970).

Doenças como diabetes, infarto do miocárdio e doenças degenerativas modificam a frequência cardíaca causando alterações no sistema cardiovascular. Dessa forma o nó sinusal entra em ação para garantir a homeostase e regular a frequência cardíaca (Mitchell, 1990).

\subsection{Problemas cardiovasculares}

A principal causa de morte no Brasil são os problemas cardiovasculares, sendo responsáveis por 30\% dos óbitos independentes de variação de idade. O que não diferencia a nível global, seguida de câncer e doenças infecciosas (Grundy et al., 1999).

Os maiores causadores de problemas cardiovasculares são o fumo, diabetes, colesterol alto, hipertensão e idade. Sendo o mais frequente a hipertensão. Além destes ainda tem a predisposição genética que também contribui bastante. Dos fatores predisponentes temos: história familiar precoce, sedentarismo, obesidade, etnia e fatores psicossociais (Grundy, 1999).

A menopausa também é um fator a ser pesquisado, já que na maioria dos casos os pacientes se encontram na idade referente a menopausa onde as mulheres geralmente estão em uma idade a cima de 55 anos e os homens a cima de 45 anos 
(Grundy, 1999).

A menopausa nada mais é que abstenção de estrogênios que combatem a aterosclerose. Por conta disso a idade influência diretamente, quanto maior a quantidade de placas ateroscleróticas, maior o risco de adquirir a doença o que aumenta de acordo com a idade. Estudos mostram que a utilização de reposição hormonal em mulheres explique a abstenção de estrogênica na aterogênese (Collins et al., 1990).

Esses fatores de riscos quando são reduzidos, diminui também a morbimortalidade secundária à aterosclerose.

Tabela 1 - Fatores de riscos em pacientes com problemas cardíacos:

\begin{tabular}{|c|c|}
\hline Redução dos fatores de riscos & Redução de chances de mortalidade \\
\hline Colesterol & $30 \%$ \\
\hline AVC & $42 \%$ \\
\hline Fumo & $70 \%$ \\
\hline
\end{tabular}

Fonte: Dados da Pesquisa (2021).

Pesquisas demonstram que houve uma queda na diminuição da morbimortalidade nos Estados Unidos nos últimos 20 anos. Porém ainda existem muitos problemas com os fatores de risco principalmente com o colesterol, obesidade e diabetes (Santos et al., 2000a).

Segundo estudo de Monteiro et al. (2000) mostram que 25\% apenas dos portadores de hipertensão arterial, estão com a doença controlada. Mas a prevalência dos portadores de doenças cardiovasculares seriam os portadores de diabetes.

Nas últimas décadas aconteceu um grande aumento na prevalência da obesidade na Europa, Estados Unidos e Brasil, o que se torna um alerta para esse fator de risco. Por esse fator trouxe um aumento também para diabetes e colesterol o levou um aumento na mortalidade (Lima et al. 2002).

O trabalho de Lima et al. (2002) mostra que com a obesidade teve um aumento de gordura na região abdominal associado a lipemia pós-prandial mesmo com triglicerídeo normal (Santos et al., 2001).

Para a real redução de problemas cardiovasculares, há uma necessidade de redução nos fatores de risco. Uma das grandes melhorias a serem utilizadas são: a melhoria na dieta, práticas de exercícios físicos, não fumar, dentre outros (Santos et al., 2000b).

\subsection{Plano de reorganização da atenção à hipertensão arterial e ao diabetes mellitus}

No Brasil, nos últimos anos, as doenças cardiovasculares vêm representando 69\% dos gastos no Sistema Único de Saúde (SUS), ela é responsável pelo aumento de frequência de internações. Em 2007 teve 1.155.489 internações por doenças cardiovasculares, com um valor global gasto de $\mathrm{R} \$ 1.466 .421 .385,12$ e um total de 91.182 mortes (DATASUS, 2007).

As modificações de rotinas também afetam a sociedade como trabalhos mais longos, onde a maioria das pessoas escolhem por lanches rápidos ou até mesmo a não realização de exercícios físicos ou também descansar por mais tempo, ter robbies como assistir televisão, jogas vídeo games, dentre outras mudanças. Tudo isso afeta a saúde da população. Nos dias de hoje também temos novos tipos de trabalhos como o de Home Office ou estudo a distância auxiliando o sedentarismo gerando a obesidade (Malta, Cezario \& Moura, 2006).

Já no ano 2000 foi gerado um plano pelo Ministério da Saúde no Brasil, o Plano de Reorganização da Atenção à Hipertensão Arterial e ao Diabetes Mellitus, com o intuito de gerar metas para organizar de forma mais clara o Sistema Único de saúde, organizando a rede básica para a triagem e acompanhamento de pacientes com diabetes e hipertensão, direcionando 
assim esses pacientes para unidades de saúde, esse trabalho também auxilia na epidemiologia das doenças (Brasil, 2004).

Outro fator que auxilia a obesidade são as condições objetivas de oferta, demanda, consumo, modismo e ainda as representações sociais da cultura e das relações sociais estabelecidas na sociedade (Buss, 2002).

\subsection{O aumento de patologias no coração com o uso de hidroxicloroquina e cloroquina}

Com a grande pandemia e o desconhecimento de medicamentos para a doença, foram utilizados medicamentos que já são utilizados para outras doenças como: antirretrovirais, antibióticos, dentre outros (Juurlink, 2020).

Segundo o estudo de Gautret et al. (2020), mostrou que nos pacientes $(n=6)$ que utilizaram a combinação de hidroxicloroquina/cloroquina unido a azitromicina tiveram como resultado a neutralização do vírus, com isso se iniciou o protocolo para a utilização dos fármacos.

A cloroquina foi um dos primeiros fármacos e ser utilizado com base nos primeiros estudos realizados para possíveis tratamentos. Contudo novos estudos mostraram que o antimalárico não possuía nenhum efeito contra o vírus e pior possuía muitos efeitos colaterais, sendo que na maioria eram relacionados ao coração (Lane et al., 2020).

Houve um grande aumento no risco de angina e insuficiência cardíaca, crescendo assim o número de óbitos em 119\% em 30 dias, devido a piora cardiovascular causada pelo efeito colateral da cloroquina e hidroxicloroquina (Borba et al., 2020).

\subsection{Nova miocardite ocasionada pelo novo vírus do COVID-19}

Durante a pandemia foi observado que o novo coronavírus além de lesionar o pulmão e levar a sequelas, foi observado que ele também afeta o coração levando a um maior número de óbitos (Kenneth Mcintosh, 2020).

Diversas pesquisas mostram que o SARS-CoV-2 está ligado a lesões miocárdica e complicações arrítmicas. De acordo com trabalhos realizados na China, mostraram que em 20 a 30\% dos pacientes hospitalizados possuíam lesões cardíacas fazendo assim com que $40 \%$ dos pacientes evoluíssem para óbitos. Algumas das anormalidades descritas foram injúria miocárdica (20\% dos casos), arritmias (16\%), miocardite (10\%), além de insuficiência cardíaca (IC) e choque (até $5 \%$ dos casos) (AHA, 2020).

Um paciente que possui diabetes ou problemas cardíacos, tem um risco duas vezes maior de falecer do que demais fatores de riscos. Com isso, devemos ter uma maior atenção com doenças cardíacas em pacientes positivos para a doença (Akhmerov \& Marbán, 2020).

Nos casos graves do novo coronavírus, internados em unidades de terapia intensiva (UTI), a hipertensão, doenças cardiovasculares/cerebrovasculares e diabetes, foram mais frequentes que outros fatores. No mínimo $8 \%$ dos pacientes positivos para a doença possuíram lesões cardíacas agudas é muito importante que possam estudar cada vez mais essas doenças e sequelas relacionadas ao coronavírus, para que os pacientes não possam evoluir a óbito (Wan et al., 2020).

\section{Conclusão}

Esse trabalho conclui que de acordo com as pesquisas existe uma grande correlação do vírus com as doenças cardiovasculares e lesões miocárdica, por conta do aumento da troponina, levando os pacientes a complicações maiores. Isso ocorre também por conta do aumento dos marcadores levando a lesões cardiovasculares.

O SARS-CoV-1 e SARS-CoV-2 atinge as células através da enzima conversora de angiotensina 2, isso provavelmente ocorre com os portadores desse vírus. Entretanto o novo Coronavírus ainda é uma doença nova e suas complicações ainda são incertas e também ainda existem muitas incertezas sobre a origem tanto da doença como de suas complicações, havendo grande necessidade de mais pesquisas. 


\section{Referências}

Aha. Statement From The American Heart Association, the Heart Failure Society of America and the American College of Cardiology. (2020). Patients taking ACE-i and ARBs who contract COVID-19 should continue treatment, unless otherwise advised by their physician. $\begin{aligned} & \text { Akhmerov, A., \& } \\ & \text { https://doi.org/10.1161/CIRCRESAHA.120.317055 }\end{aligned}$ Marbán, E. (2020). COVID-19 and the Heart. Circulation research, $126(10), \quad 1443-1455$.

Brasil. Organização Pan-Americana da Saúde (OPAS). (2004). Avaliação do Plano de Reorganização da Atenção à Hipertensão Arterial e ao Diabetes Mellitus no Brasil. Brasília: Ministério da Saúde (MS).

Borba, M., Val, F., Sampaio, V. S., Alexandre, M., et al. CloroCovid-19 Team (2020). Effect of High vs Low Doses of Chloroquine Diphosphate as Adjunctive Therapy for Patients Hospitalized With Severe Acute Respiratory Syndrome Coronavirus 2 (SARS-CoV-2) Infection: A Randomized Clinical Trial. JAMA network open, 3(4), e208857. https://doi.org/10.1001/jamanetworkopen.2020.8857

Buss, P. M. (2002). Promoção da Saúde da Família. Rev Bras. Saúde Família; 2(6):50-63.

Collins, R., Peto, R., Macmahon, S., et al. (1990). Blood pressure, stroke, and coronary heart disease. Part 2: Short-term reductions in blood pressure: overview of randomized drug trials in their epidemiological context. Lancet; 335:827-38.

Datasus. (2007). [homepage na internet]. Indicadores e dados básicos - Brasil.

Driggin, E., Madhavan, M. V., Bikdeli, B., Chuich, T., Laracy, J., Bondi-Zoccai, G., et al. (2020). Cardiovascular Considerations for Patients, Health Care Workers, and Health Systems During the Coronavirus Disease 2019 (COVID-19) Pandemic. J Am Coll Cardiol.

Ewing, D. J., Marten, C. N., Young, R. J., Clarke, B. F. (1985). The value of cardiovascular autonomic function tests: 10 years experience in diabets. Diabets Care; 8:491-8.

Fang, L., Karakiulakis, G., Roth, M. (2020). Are patients with hypertension and diabetes mellitus at increased risk for COVID-19 infection? Lancet Respir Med; $8: \mathrm{e} 21$

FioCruz - Fundacao Oswaldo Cruz. (2020). [homepage nainternet]. Covid-19: Fiocruz Amazonia conclui o primeiro sequenciamento do vírus na região Norte.

Gamelin, F. X., Berthoin, S., Bosquet, L. (2006). Validity of the polar S810 heart rate monitor to measure R-R intervals at rest. Med Sci Sports Exerc; 38(5):887-93.

Gautret, P., Lagier, J. C., Parola, P., et al. (2020). Hydroxychloroquine and azithromycin as a treatment of COVID-19: results of an open-label nonrandomized clinical trial. International journal of antimicrobial agents. 105949.

Grundy, S. M., Pasternak, R., Greenland, P., Smith Jr, S., Fuster, V. (1999). Assessment of cardiovascular risk by use of multiplerisk- factor assessment equations: A statement for healthcare professionals from the American Heart Association and the American College of Cardiology. Circulation; 100:1481-92.

Grundy, S. M. (1999). Age as a risk factor: you are old as your arteries. Am J Cardiol; 83:1455-7.

Guyton, A. C., \& Hall, J. E. (2006). Tratado de Fisiologia Médica. Elsevier edição 11.

Hon, E. H., \& Lee, S. T. (1965). Electronic evaluations of the fetal heart rate patterns preceding fetal death. Am J Obstet Gynecol; 87:817-26.

Junqueira Jr, L. F. (1990). Sobre o possível papel da disfunção autonômica cardíaca na morte súbita associada à doença de Chagas. Arq Bras Cardiol; 56(6):429-34.

Juurlink, D. N. (2020). Safety considerations with chloroquine, hydroxychloroquine and azithromycin in the management of SARS-CoV-2 infection. CMAJ.

Kauark, F. (2010). Metodologia da pesquisa: guia prático / Fabiana Kauark, Fernanda Castro Manhães e Carlos Henrique Medeiros. - Ita- buna: Via Litterarum editora, $88 \mathrm{p}$.

Kenneth Mcintosh, M. D. (2020). Coronavirus disease 2019 (COVID-19). Available at: https://www. uptodate.com/contents/coronavirus-disease-2019-covid19 Respiratory Syndrome Coronavirus 2 (SARS-CoV-2) Infection: A Randomized Clinical Trial. JAMA network open; 3(4): e208857.

Kim, Y., Kwon, O., Paek, J. H., et al. (2020). Two distinct cases with COVID-19 in kidney transplant recipients. American journal of transplantation: official journal of the American Society of Transplantation and the American Society of Transplant Surgeons.

Lane, et al. (2020). Safety of hydroxychloroquine, alone and in combination with azithromycin, in light of rapid wide-spread use for COVID-19: $a$ multinational, network cohort and self-controlled case series study.

Leon, D. F, Shaver, L. A., \& Leonard, J. J. (1970). Reflex heart rate control in man. Am heart 2. J; 80:729-39.

Lima, J. G., Nóbrega, L. H. C., Nóbrega, M. L. C., Bandeira, F., \& Souza, A. G. P. (2002). Dislipidemia pós-prandial como achado precoce em indivíduos com baixo risco cardiovascular. Arq Bras Endocrinol Metab; 46(3): 249-254.

Malta, D. C., Cezario, A. C., \& Moura, L. (2006). A construção da vigilância e prevenção das doenças crônicas não transmissíveis no contexto do Sistema Único de Saúde. Epidemiol Serv Saúde; 15(3): 47-65

Marães, V. R., Silva, E., Catai, A. M., Novais, L. D., Moura, M. S., Oliveira, L., et al. (2005). Identification of anaerobic threshold using heart rate response during dynamic exercise. Braz J Med Biol Res; 38(5): 731-5. 
Research, Society and Development, v. 10, n. 7, e53210716590, 2021

(CC BY 4.0) | ISSN 2525-3409 | DOI: http://dx.doi.org/10.33448/rsd-v10i7.16590

Million, M. (2020). Early treatment of COVID-19 patients with hydroxychloroquine and azithromycin: A retrospective analysis of 1061 cases in Marseille, France. Travel medicine and infectious disease.

Mitchell, J. J. B. (1990). Wolffe memorial lecture. Neural control of the circulation 3. during exercise. Med Sci Sports Exerc; 22(2): 141-54.

Monteiro, C. A., D’A Benicio, M. H., Conde, W. L., \& Popkin, B. M. (2000). Shifting obesity trends in Brazil. Eur J Clin Nutr; 54:342-6.

Opas. Organizacao Pan-Americana Da Saude (2020). [homepage na internet]. OMS afirma que COVID-19 e agora caracterizada como pandemia.

Santos, R. D., Spósito, A. C., Santos, J. E, et al. (2000). Programa de avaliação nacional do conhecimento sobre prevenção da aterosclerose (PANDORA): Como tem sido feito o tratamento das dislipidemias pelos médicos brasileiros. Arq Bras Cardiol; 75:289-95. A.

Santos, R. D., Sposito, A. C., Ventura, L. I., César, L. A. M., Ramires, J. A. F., \& Maranhão, R. C. (2000). Pravastatin increases the plasma removal of chylomicron-like emulsions in men with coronary artery disease. Am J Cardiol; 85:1163-6. B.

Santos, R. D., Ventura, L. I., Spósito, A. C., Schreiber, R., Ramires, J. A. F., \& Maranhão, R. C. (2001). The effects of gemfibrozil upon the metabolism of chylomicron-like emulsions in patients with endogenous hypertriglyceridemia. Cardiovasc Res; 49:456-65.

Wan Y, Shang J, Graham R, et al. (2020). Receptor Recognition by the Novel Coronavirus from Wuhan: an Analysis Based on Decade-Long Structural Studies of SARS Coronavirus. J Virol; 94

Zheng, Y. Y., Ma, Y. T, Zhang, J. Y., \& Xie, X. (2020). COVID-19 and the cardiovascular system. Nat Rev Cardiol. 